\author{
ANNALS OF THE \\ UNIVERSITY OF CRAIOVA
}

Series: $\checkmark$ Biology

$\checkmark$ Horticulture

$\checkmark$ Food products processing technology

$\checkmark$ Environmental engineering

Vol. XXVI (LXII) - 2021

\title{
FLORISTIC AND VEGETATION STUDIES IN THE CELARU AREA, DOLJ COUNTY. MEASURES FOR THE CONSERVATION OF NATURAL VEGETATION
}

\author{
Răduțoiu Daniel ${ }^{1 *}$, Mitroi Viorel Ionuț ${ }^{2}$ \\ ${ }^{1 *}$ University of Craiova, Faculty of Horticulture \\ ${ }^{2}$ University of Craiova, Faculty of Horticulture, Craiova, Student \\ * Correspondence author. E-mail: radutoiudaniel@yahoo.com
}

Keywords: Celaru, conservation, flora, vegetation,

\begin{abstract}
The commune of Celaru, with the administrative center in the homonym village, is made up of Ghizdăvești, Soreni, Celaru, and Marotinu de Jos settlements. The main asset of the local administrative unit is the fertile land that is cultivated with wheat, corn, sunflower, rapeseed (along with crops such as sugar beet, hemp, flax, which were common in the past), as well as the large areas covered by black locust plantations that are a strong barrier in the way of sand dunes, which extend all the way from the Jiu river.

Al. Buia, M. Păun, and Gh. Popescu have provided sporadic data on the spontaneous flora from the area under study. The analysis of the flora identified on the surface of Celaru commune highlights the xerophilous character of the area. Approximately $3 / 4$ of the spontaneous flora characteristic to Celaru settlement is adapted to a prolonged water stress. The vegetation that grows on the territory of this commune and on its borderline areas is subject to a strong zoo-anthropogenic impact. The natural vegetation is almost absent, the semi-natural one covers small areas, and the anthropogenic one is encountered at the edge of crops and of ruderal surfaces, as well as at the level of black locust plantations.
\end{abstract}

\section{INTRODUCTION}

A brief look at a more distant past is necessary in order to better understand the variability of the flora within the area under study.

Several taxa from the area are mentioned in the study entitled "Conspectul Florei României" (D. Grecescu, 1898). The authors of the present paper also found most of them, with few exceptions. Numerous data were also inserted in the monumental work entitled "Flora R.P.R.-R.S.R. " (vol. I-XII).

Al. Buia, M. Păun, and Gh. Popescu have provided data about the spontaneous flora from the area under study.

Significant studies on the flora and vegetation of certain areas that share physical-geographical similarities with the territory researched by us in the present paper were carried out by the famous botanists of the Craiova university center (Buia \& Popescu 1952, Buia 1959, Buia \& Păun 1960, Buia et al. 1961, Păun 1964; Popescu 1974; Maloş \& al. 1972, Păun 1963, 1966, 1967, 1979), Păun \& Popescu 1972, 1975; Păun \& al. 1972, Popescu \& Costache 1999; Costache 2005; Răduţoiu 2008 etc.). 
A special mention is attributed to the elaboration of the well-known herbarium work "Flora Olteniae Exiccata", initiated in Craiova by professor Al. Buia and continued under the leadership of professor M. Păun, which includes cited taxa from the territory under study.

\section{MATERIAL AND METHODS}

Our research was conducted on the territory of Celaru settlement and on its surroundings. This settlement is located on the DJ 604 County Road, along the Leu - Zvorsca - Vișina roadway. The first village of the commune is Ghizdăvești, followed by Soreni, then Celaru, which also represents the administrative center of the commune, while the succeeding villages are Marotinu de Jos and Marotinu de Sus (Ancuța et al., 2009). In the framework of Dolj county, the territory under study is located in the south-eastern part of the administrative unit (Fig. 1). The borders of Celaru local administrative unit are marked by Apele-Vii and Mârșani communes towards west, by Amărăștii de Sus commune towards the south, by Dioști commune towards the north, and by Redea commune towards the east.

The Celaru administrative unit covers a total area of 9,748 hectares, including an agricultural surface of 7,834 hectares, out of which $98.7 \%$ is represented by arable land, $1.2 \%$ by vineyards and grapevine nurseries, and $0.1 \%$ by orchards and associated nurseries.

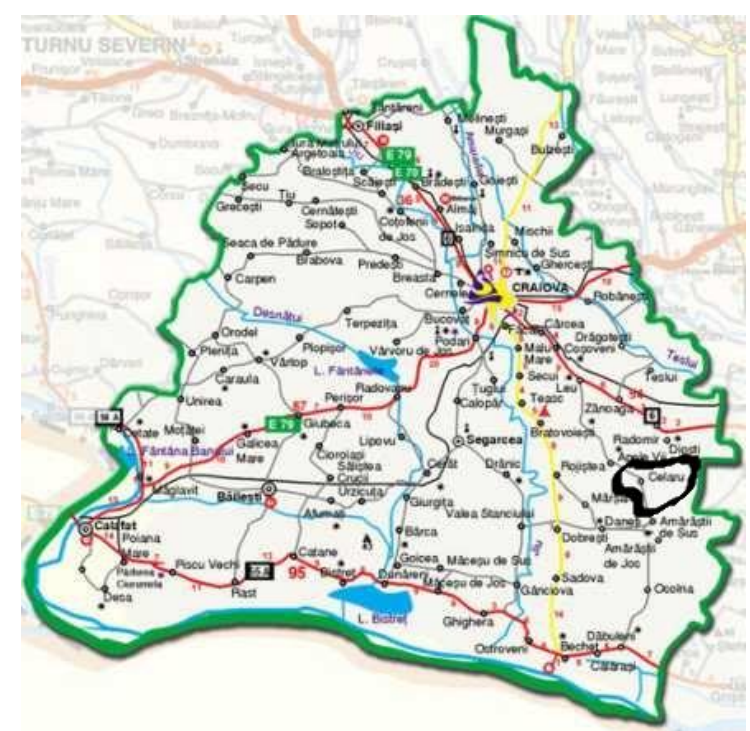

Figure 1. Location and delimitation of the researched territory within Dolj county (http://www.profudegeogra.eu/wp-content/uploads/2011/05/Harta-administrativa-ajudetului-Dolj.jpg)

The entire administrative territory of Celaru commune falls into the Leu Rotunda high field, which appears as a long, elevated section originating in the Getic Plateau and extending to the south, towards the Oltenia Plain.

The absolute altitude of the field is comprised between 110 and 138 meters and it decreases from northwest to southeast, as well as from west to east. 
From a geological point of view, at the base there are to be found Levantine fluvial - lacustrine deposits consisting of clays, marl, sands and covered by Quaternary deposits formed by loess, red clays, and aeolian sands, located in sand dunes.

Geomorphologically, it is located in the high plain from the south of Oltenia, on mobile sands consolidated by black locust plantations, with small depression areas between the sand dunes. The water table rises to $4-12$ meters, with positive or negative changes occurring at an interval comprised between $25-30$ years (Ancuța et al. 2009).

The area has groundwater resources that require priority measures in order be protected against pollution, the water table being affected by nitrites. The territory of the administrative unit is not marked by the presence of flowing or stagnant waters.

The following soil units and groups are characteristic for the area under study: moderately-textured medium steppe brown chernozems, medium-textured forest-steppe leached chernozems, slightly fallow and leached sands and sandy soils, and light-textured reddish brown forest soil.

The first step in the achievement of the floristic inventory of Celaru administrative unit was represented by the examination of the specialized literature regarding the studies previously conducted on this part of Oltenia region.

Some publications inserted in the reference list represented a material of particular importance, due to the resemblance of the geographical area that they cover with the one corresponding to the territory under study in the present paper.

After the documentation, field studies were repeatedly performed in order to observe, analyze and collect the floristic material.

The identification of the taxa was conducted on fresh material or on material preserved in dry state, by using different sources of taxonomic information (Săvulescu T. \& al. 1952 -1976; Beldie Al. 1977, 1979; Ciocârlan V. 2009, Sârbu et al. 2013 and others).

In the study of vegetation, surveys were carried out in the characteristic areas. All the species found within these areas were registered, along with the station characteristics. The delineation of the associations was achieved following the processing of field data. Due to the fact that the identified associations are known in the field literature, only their local characteristics are presented in the present paper.

\section{The flora}

\section{RESULTS AND DISCUSSIONS}

The floristic studies on the Celaru area and on its surroundings highlight the presence of numerous vascular species. In the following synopsis, these species have been arranged in a systematic order, by botanical families.

Phyl. Spermatophyta, Cl. Magnoliopsida, Fam. Aristolochiaceae: Aristolochia clematitis L. - G., $2 n=14$., xeromez., Centr. eur. SV; Fam. Ranunculaceae: Nigella arvensis L. - T., $2 \mathrm{n}=12$., mezoxer., Pont.-medit.; Consolida regalis S.F. Gray - T., $2 n=16$., mezoxer., Eur.; Clematis vitalba L. - Ph., $2 n=16$., mez., Eur. Centr.; Ranunculus arvensis L. - T., $2 \mathrm{n}=32$., mez.-mezoxer., Euras.; $R$. bulbosus L. - H.(G.), 2n=16., Centr. and N. Eur.; R. sardous Cr. - T., $2 \mathrm{n}=16$., mezohigr., Eur.; R. repens L. - H., $2 \mathrm{n}=32$., mezohigr.- higr., Euras.; Adonis aestivalis L. - T., $2 \mathrm{n}=32$., mezoxer., Euras. Cont.; Fam. Papaveraceae: Papaver rhoeas L. T., 2n=14., mezoxer., Cosm.; P. dubium L. - T., 2n=42., xeromez., Eur.; Chelidonium majus L. - H., $2 \mathrm{n}=12$., mez., Euras.; Fam. Fumariaceae: Fumaria schleicheri Soy.Willem - T., 2n=32., mezoxer., Euras.; F. vaillantii Loisel. - T., $2 \mathrm{n}=32$., xer.-mez 
Euras.; F. rostellata Knaf. - T., $2 \mathrm{n}=14$., xeromez., Centr. eur.-balc.; Fam. Ulmaceae: Ulmus minor Mill. - Ph., $2 \mathrm{n}=28$., mezoxer., Eur.; Fam. Moraceae: Morus alba L. Ph., 2n=28., „Dabului road”, xer., China; Fam. Cannabaceae: Cannabis sativa subsp. spontanea (Vavilov) Serebr. - T., 2n=20., xer., Cont. Euras., Humulus lupulus L. - H., $2 n=20$., mezohigr., Euras., North Am.; Fam. Urticaceae: Urtica urens L. - T., 2n=24, 26, 52., mez., „Caracal road”, Cosm., U. dioica L. - H., 2n=26, 48, 52., mez.mezohigr., „Caracal road”, Cosm.; Fam. Phytolaccaceae: Phytolacca americana L. H., 2n=36, xer., „La grinduri”, Am. de N.; Fam. Molluginaceae: Mollugo cerviana (L.) Ser. - T., xer., „Dabului road”, Medit.; Fam. Portulacaceae: Portulaca oleracea L. T., $2 n=54$., xer., Cosm.; Fam. Caryophyllaceae: Scleranthus annuus L. - T., $2 n=44$., xer., Euras., Arenaria serpyllifolia L. - T., $2 n=40$., xer.-xeromez., „Dabului road”, Circ.; Holosteum umbellatum L. - T., 2n=20., xer., „Delniță”, Euras., Stellaria media (L.) Vill. - T.-Ht., $2 \mathrm{n}=40,42$, 44., mez., Cosm., Cerastium glomeratum Thuill. - T., $2 \mathrm{n}=72$., mezohigr., Cosm., C. brachypetalum Pers. - T., $2 \mathrm{n}=90$, mezoxer. Euras., Gypshophila muralis L. - T., $2 \mathrm{n}=34$., mez.-xeromez., Euras., Kohlrauschia prolifera (L.) Kunth - T., 2n=30., xer., Atl.-medit., Silene borysthenica (Gruner) Walters - Ht.H., $2 n=24$., xer., „Dabului road”, Euras. Cont., S. vulgaris (Moench) Garcke - H., $2 \mathrm{n}=24$., xer., Euras., Spergula arvensis L. - T., $2 \mathrm{n}=18$., mez., Cosm.; Fam. Amaranthaceae: Amaranthus albus L. - T., $2 \mathrm{n}=32$., xer.- mezoxer., Adv. (North Am.), A. powellii S. Watson - T., $2 n=32$., xeromez., Adv. (North Am.)., A. retroflexus L. - T., 2n=32,34., xeromez.-mez., Adv. (North Am.).; Fam. Chenopodiaceae: Chenopodium album L. - T., $2 n=36,54 .$, mez., Cosm., Atriplex tatarica L. - T., $2 \mathrm{n}=18$., mezoxer., Euras., A. patula L. - T., $2 \mathrm{n}=36$., Circ., Bassia scoparia (L.) Voss - T., $2 n=18$., xer., Euras., Salsola kali subsp. ruthenica L. - T., $2 n=36$., xeromez., „Dabului road”, Euras.; Fam. Polygonaceae: Polygonum arenarium Waldst. et Kit. T., $2 \mathrm{n}=40$., mezohigr., Pont.-pan.-medit.; $P$. aviculare $\mathrm{L}$. - T., $2 \mathrm{n}=60$., mezohigr., Cosm., P. convolvulus (L.) - T., $2 \mathrm{n}=40$., xeromez., Circ., Rumex sanguineus L. - H., $2 \mathrm{n}=20$., mezohigr., „La grinduri”, Eur.; $R$. crispus L. - H., $2 n=60$., mez.- mezohigr., Euras.; Fam. Rosaceae: Rubus candicans - Ph., $2 \mathrm{n}=21$., mezohigr., Eur. centr. and V.; Fragaria viridis (Duchesne) Weston - H., $2 n=14$., xeromez., Euras., Potentilla argentea L. - H., xeromez., $2 n=14$., „La grinduri”, Euras., P. recta L. - H., $2 n=28,42$. , xeromez., Cont. Euras., Geum urbanum L. - H., 2n=42., mez.-mezohigr., Circ., Rosa canina L. s.l. - Ph., $2 n=35$., xeromez.-mez., „Dabului road”, Eur., Crataegus monogyna Jacq. - Ph., $2 \mathrm{n}=34$., mezoxer.-mez., Eur., Prunus spinosa L. - Ph., 2n=32., xeromez.-mez., „Drumul Dabului”, Eur., P. cerasifera Ehrh. - Ph., $2 n=16$., xeromez., „Dabului road”, Pont.-balc.; Fam. Caesalpinaceae: Gleditsia triacanthos L. - Ph., 2n=28., xer., Am. de N.; Fam. Fabaceae: Medicago lupulina L. - T.-H., $2 \mathrm{n}=16$, 32., xeromez.-mezohigr., Euras., M. minima (L.) - T., $2 \mathrm{n}=16$., xer.-xeromez., Submedit., Trifolium arvense L. - T., $2 \mathrm{n}=14$., xeromez., Euras., T. campestre Schreb. - T., $2 n=14$., xeromez.-mez., Eur., Lotus corniculatus L. - H., $2 n=24$., xer., Euras., Amorpha fruticosa $\mathrm{L}$. - Ph., $2 \mathrm{n}=40$., xer., North Am., Robinia pseudoacacia L. - Ph., $2 n=20,22$., xer., North Am., Vicia grandiflora Scop. - T., $2 n=12$, 14., xeromez., Pont.balc.-cauc., $V$. sepium L. - H., $2 n=14$., mez.-mezohigr., „La grinduri”, Euras., $V$. villosa Roth - T.-Ht., $2 n=14$., xeromez., Eur., V. pannonica Crantz - T., $2 n=12$., xeromez.-mez., Pont.-medit., Lathyrus tuberosus L. - H., $2 n=14$., xeromez.-mez., Euras.; Fam. Onagraceae: Circaea lutetiana L. - G., $2 n=22$., mezohigr., Euras.medit.; Fam. Elaeagnaceae: Elaegnus angustifolia L. - Ph., $2 \mathrm{n}=28$., xeromez., "Dabului road”, Temp. As.; Fam. Cornaceae: Cornus sanguinea L. - Ph., 2n=22., mez.-mezohigr., Eur. Centr.; Fam. Euphorbiaceae: Euphorbia helioscopia L. - T., 
$2 \mathrm{n}=42$., xeromez.-mez., Euras., E. cyparissias L. - H., $2 \mathrm{n}=20$, 40., xeromez., Euras., Chamaesyce maculata (L.) Small. - T., $2 n=28$., xeromez., Adv. North Am.; Fam. Simaroubaceae: Ailanthus altissima (Mill.) Swingle - Ph., $2 \mathrm{n}=64$., „La grinduri”, China.; Fam. Zygophyllaceae: Tribulus terrestris L. - T., 2n=12, 24, 36, 48., xer. xeromez., „La grinduri”, Centr.-eur.-medit.; Fam. Oxalidaceae: Oxalis corniculata L. - T.-H., 2n=24, 44, 48., mez., Adv. (Medit.)., O. fontana Bunge. - H., 2n=24., mez., Adv. (North Am.).; Fam. Geraniaceae: Geranium pusillum Burm. f. - T., 2n=26, 34, 36., xeromez.-mez., „La grinduri”, Eur., G. dissectum L. - T., $2 \mathrm{n}=22$., xeromez.-mez., Euras., Erodium cicutarium (L.) L'Herit. - T., 2n=40., xeromez., „La grinduri”, Cosm.; Fam. Apiaceae: Eryngium campestre L. - H., 2n=14, 28., xer.- xeromez., „La grinduri", Pont.-medit., Anthriscus cerefolium subsp. trichosperma (L.) Hoffm. - T., 2n=18., mez., „La grinduri”, Pont.-medit., Daucus carota L. - Ht., $2 \mathrm{n}=18$., xer., Euras., Falcaria vulgaris Bernh. - Ht.(T.,H.), 2n=22., xer.-xeromez,, Euras. (submedit.)., Tordylium maximum L. - T.-Ht., $2 \mathrm{n}=22$., xer.-xeromez. Eur. centr. and S, As. SV.; Fam. Hypericaceae: Hypericum perforatum L . - H., $2 n=32$., xeromez.-mez., Euras.; Fam. Malvaceae: Malva sylvestris L. - Ht.-H., $2 \mathrm{n}=42$., xeromez.-mez., Euras. , Abutilon theophrasti Medik. - T., 2n=42., mez.-mezohigr., Euras., Hibiscus trionum L. - T., $2 n=28,56$., xeromez.-mez., Euras.; Fam. Violaceae: Viola arvensis Murray. - T., $2 n=34 .$, xeromez.-mez., Cosm., V. kitaibeliana Schult. - T., $2 n=16$, 48., xer.xeromez., „La grinduri”, Pont.-medit., V. odorata L. - H., 2n=20., mez., Atl.-medit.; Fam. Brassicaceae: Sisymbrium officinale (L.) Scop. - T.-Ht., $2 \mathrm{n}=14$., xeromez.mez., Euras., S. altissimum L. - T.-Ht., $2 \mathrm{n}=14$., xeromez., Euras. Cont., S. orientale L. - T., Ht., $2 \mathrm{n}=14$., xer.-xeromez., Pont.-medit., Descurainia sophia (L.) Webb ex Prantl - T.-Ht., 2n=28., xeromez.-mez., Euras., Arabidopsis thaliana (L.) Heynh. - T.Ht., $2 \mathrm{n}=10$., xeromez., „Dabului road”, Cosm., Berteroa incana (L.). DC. - Ht., 2n=16., xeromez.-mez., „Dabului road”, Euras., Erophila verna (L.) Chevall. - T., xeromez., $2 \mathrm{n}=$....., Eur., Cardamine pratensis L. - H., $2 \mathrm{n}=16$., mezohigr.-higr., „Caracal road”., Capsella bursa-pastoris (L.) Medik. - T.-Ht., $2 \mathrm{n}=32$., xer.-xeromez., „Delniță, Cosm., Thlaspi arvense L. - T.-Ht., $2 \mathrm{n}=14$., xeromez.-mez., „Delniță”, Euras., T. perfoliatum L. - T.-Ht., $2 \mathrm{n}=14,42,70$. , xeromez.-mez., Euras., Cardaria draba (L.) Desv. - H., $2 n=62,64 .$, xeromez., „Delniță”, Euras. Medit., Lepidium campestre (L.) R. Br. - T.Ht., $2 \mathrm{n}=16$., xeromez.-mez., Eur., L. ruderale L. - T.-Ht., $2 \mathrm{n}=16$, 28, 32., xeromez.mez., Euras., Diplotaxis tenuifolia (L.) DC. - H.(Ch.), $2 n=22$. , xer.- xeromez., Centr. eur.-medit., Sinapis arvensis L. - T., $2 n=18 .$, mez.-xeromez., Euras., Calepina irregularis (Asso) Thell. - T.-Ht., 2n=28., xeromez., Pont.-medit.; Fam. Resedaceae: Reseda lutea L. - Ht.-H., 2n=24, 48., xeromez., Euras.; Fam. Salicaceae: Salix fragilis L. - Ph., higr., $2 n=76$., „Dabului road”, Euras.; Fam. Primulaceae: Anagallis arvensis L. - T.-Ht., $2 \mathrm{n}=40$., mez., Circ.; Fam. Convolvulaceae: Calystegia sepium (L.) R.Br. - G.(H.), 2n=22, 24., mezohigr.-higr., Euras., Convulvulus arvensis L. (G.)H., 2n=50., mez., Cosm.; Fam. Cuscutaceae: Cuscuta campestris Yuncker - T., $2 n=56$., xeromez.-mez., Adv. (North Am.)., C. europaea L. - T., $2 n=14$., xeromez.mez., Euras., C. epithymum (L.) L - T., $2 n=14$., xeromez.-mez., Euras.; Fam. Boraginaceae: Lithospermum arvense L. - T., $2 \mathrm{n}=16,24$, 28., xeromez.-mez., Euras., Echium italicum L. - Ht., $2 \mathrm{n}=16$, 32., xeromez., Euras. de S., E. vulgare L. Ht., $2 \mathrm{n}=16$, 32., xeromez., „La grinduri”, Euras., Symphytum officinale L. s. str. - H., $2 \mathrm{n}=36,48$., mezohigr., Euras., Anchusa officinalis L. - H.(Ht.), $2 \mathrm{n}=16$., xer.-xeromez., Eur., Lappula squarrosa (Retz.) Dumort. - T.-Ht., $2 \mathrm{n}=48$., xeromez., Euras., Asperugo procumbens L. - T., 2n=48., xeromez., Euras. Cont., Cynoglossum officinale L. - Ht., $2 n=24$., xeromez., Euras. Cont.; Fam. Verbenaceae: Verbena 
officinalis L. - Th.-H., 2n=14. xeromez.-mez., Cosm.; Fam. Lamiaceae: Ajuga genevensis L. - H., 2n=32., xeromez.-mez., Euras., Marrubium vulgare L. - H., $2 \mathrm{n}=34$., xeromez., Euras., Marrubium peregrimun L. - H., 2n=34., xeromez., Pont.pan.-balc., Glechoma hirsuta Waldst. et Kit. - H.(Ch.), $2 n=36$. ., mez., Pont.-medit.centr., Eur., Lamium purpureum L. - T.-Th., $2 \mathrm{n}=18 .$, xeromez.-mez., Euras., L. amplexicaule L. - Th., $2 \mathrm{n}=18$., xeromez.-mez., „Caracal road”, Euras., Ballota nigra L. - H., 2n=22., xeromez.-mez., Eur. centr. şi N-E., Salvia nemorosa L. - H., 2n=14, xer.-xeromez., Pont.-medit.-centr., Eur., Clinopodium vulgare L. - H., $2 n=20$., xeromez.-mez., Circ., Thymus pannonicus All. - Ch., $2 n=28$. , xer.- xeromez., Pont.pan., Mentha pulegium L. - H., 2n=20., mezohigr. Euras. (submedit.)., M. Iongifolia L. - H., 2n=24., mezohigr.-higr., Euras.; Fam. Plantaginaceae: Plantago lanceolata L. - H., $2 n=12$., xer., Euras., P.scabra Moench - T., $2 n=12$., xer., Cont. Euras.; Fam. Scrophulariaceae: Verbascum phlomoides L. - Ht., 2n=32, 34., xeromez.-mez., „Delniță”, Eur. centr. şi de SE., V. chaixii Vill. - H.,. 2n=26, 36., xer.-xeromez., Medit., Linaria vulgaris Mill. - H., $2 n=12$., xer.,xer., Euras., Kickxia elatine (L.) - T., $2 n=18$, 36., mez., Centr. eur.-medit., Veronica hederifolia L. - T., $2 n=54$., mez., „Caracal road”, Euras., V. persica Poir. - T., $2 n=28 .$, mez., "Dabului road”, Adv. (SV As.)., V. triphyllos L. - T., 2n=14., xeromez., „La Grinduri”, Euras., V. arvensis L. - T., $2 n=16$., xeromez.-mez., Euras., Melampyrum arvense L. - T., $2 \mathrm{n}=18$., xeromez.-mez., Eur.; Fam. Solanaceae: Lycium barbarum L. - Ph., 2n=24., xer.-xeromez., China., Hyoscyamus niger L. - Ht., 2n=34., xeromez.-mez., Euras., Solanum nigrum L. - T., $2 n=72$., mez., Cosm., Datura stramonium L. - T., $2 n=24$., xeromez.-mez., Adv. from North Am., today Cosm.; Fam. Rubiaceae: Galium aparine L. - T., $2 n=42,44,66$, 88., mez.-mezohigr., „La grinduri”, Circ., G. verum L. - H., 2n=22, 44., xeromez.mez., Euras., Cruciata pedemontana (Bellardi) Ehrend. - T., $2 \mathrm{n}=18 .$, xeromez., „La grinduri", Submedit., C. glabra (L.) Ehrend. - H., 2n=22, 44., mez., Euras., C. laevipes Opiz - H., 2n=22., xeromez.-mez., Euras.; Fam. Valerianaceae: Valerianella locusta (L.) Laterr. em. Betcke - T., $2 n=14,16 .$, xeromez.-mez., Eur., V. dentata (L.) Pollich - T., $2 n=14,16$., xeromez., Eur.; Fam. Dipsacaceae: Dipsacus fullonum L. Ht., $2 n=16$, 18., mez., Submedit., Cephalaria transylvanica (L.) Roem. et Schult. Ht., $2 n=18 .$, xer.-xeromez., Pont.-medit., Scabiosa ochroleuca L. - Ht. - H., 2n=16., Sp. xeromez., Euras. Cont.; Fam. Asteraceae: Conyza canadensis (L.) Cronquist T., $2 n=18$., xeromez.-mez., Adv. (North Am.)., Erigeron annuus (L.) Pers. - T., Ht., H., $2 n=27,36$., mez., Adv. (North Am.), Filago vulgaris Lam. - T., $2 n=28$., xeromez., Euras. de S., F. arvensis L. - T., $2 \mathrm{n}=28$., xeromez., Euras. de S., Inula britannica L. - Ht., $2 n=32$., mez.- mezohigr., Euras., I. germanica L. - H., $2 n=16$., xeromez., Centr. and SE. Eur., Pulicaria vulgaris Gaertn. - T., $2 n=18$., mezohigr., Euras., Ambrosia artemisiifolia L. - T., 2n=36., xer., Adv. (North Am.)., Xanthium spinosum L. - T., $2 \mathrm{n}=36$., xeromez.-mez., Adv. (South Am.), today Cosm., X. italicum Moretti - T., $2 \mathrm{n}=36$., mez.-mezohigr., Eur. de S., Galinsoga parviflora Cav. - T., $2 \mathrm{n}=16$., mez., Adv. (South Am. - Peru), today Cosm., G. ciliata (Rafin.) Blake - T., 2n=32., mez., Adv. (South Am.)., Anthemis cotula L. - T., 2n=18., xeromez.-mez., Cosm., A. austriaca Jacq. - T., $2 n=18$., xeromez., Centr. eur.-pont., Achillea setacea Waldst. et Kit. - H., $2 n=18$., xer.-xeromez., Euras. Cont., Matricaria recutita L. - T., $2 n=18$., xeromez.-mez., Euras., M. perforata Merat - T.-Ht., $2 \mathrm{n}=18$, 36., mez., Euras., Artemisia annua L. - T., $2 \mathrm{n}=18$., xeromez.-mez., Euras. Cont., A. absinthium L. H.(Ch.), $2 n=18$., xeromez.-mez., Euras., A. vulgaris L. - H., $2 n=18$. , mez., Circ., Tussilago farfara L. - G., $2 \mathrm{n}=60$., mez.- mezohigr., „Caracal road”, Euras., Senecio vernalis Waldst. et Kit. - T., $2 \mathrm{n}=40$., xeromez., „Dabului road”, Euras. Cont., $S$. 
vulgaris $\mathrm{L}$. - T., $2 \mathrm{n}=40$. mez., Euras., S. erucifolius L. - H., $2 \mathrm{n}=40$., xeromez.-mez., Euras., Xeranthemum cylindraceum Sibth. et Sm. - T., 2n=20., xer.-xeromez., „Dabului road”, Pont.-medit., Arctium lappa L. - Ht., $2 \mathrm{n}=36$., xeromez.-mezohigr., Euras., Carduus acanthoides L. - Ht., 2n=20, 22., xeromez., "La grinduri”, Eur., Cirsium vulgare (Savi) Ten. - Ht., $2 \mathrm{n}=68,102$. , xeromez.-mez., Euras., Onopordum acanthium L. - Ht., 2n=34., xeromez., „La grinduri”, Euras., Centaurea micranthos S.G. Gmel. ex Hayek - Ht.-H., $2 n=36$., xer., Pont.-pan.-balc., Carthamus lanatus L. T., $2 n=44$., xeromez., Pont.-medit., Picris hieracioides $\mathrm{L}-\mathrm{Ht}$. $-\mathrm{H}$., $2 \mathrm{n}=10$., xeromez.mez., Euras., Chondrilla juncea L. - Ht.-H., 2n=15, 30., xer.-xeromez., Euras., Taraxacum officinale Weber ex F.H.Wigg. - H., 2n=24., xeromez.-mez., Euras., Lactuca serriola L. - Ht., $2 \mathrm{n}=18$., xeromez.-mez., Euras., Sonchus arvensis L. - G., $2 n=36,54$., mez., Euras., Crepis foetida subsp. rhoeadifolia (M. Bieb.) Celak L. - T., $2 \mathrm{n}=10$., xeromez.-mez., Pont.-medit.; Fam. Liliaceae: Gagea villosa (M. Bieb.) Sweet - G., 2n=48., xeromez.-mez, „La Grinduri”, Eur., G. lutea (L.) Ker Gawl. - G., $2 n=72$., mez., Euras., Polygonatum latifolium (Jacq.) Desf. - G., $2 n=20$., mez., Pont.pan.-balc.; Fam. Juncaceae: Juncus effusus L. - H., $2 n=40$, 42., mezohigr., Cosm., J. inflexus L. - H., $2 \mathrm{n}=40$, 42., mezohigr., Euras.; Fam. Poaceae: Lolium perenne L. - H., 2n=14., mez., Cosm., Vulpia myuros (L.) C.C.Gmel.- T., Ht., 2n=42., xeromez., Euras. (Cosm.)., Poa bulbosa L. - H., 2n=14, 21, 28, 32, 35, 39, 40, 42, 45, 56, 58., xeromez., „Delniță”, Euras., P. annua L. - T.-H., 2n=28., mez., Cosm., P. angustifolia L. - H., 2n=46, 72., xeromez.-mez., Euras., Sclerochoa dura (L.) Beauv. - T., 2n=14., xeromez., Medit., Dactylis glomerata L. - H., 2n=28., xeromez.-mez., Euras., Apera spica-venti (L.) Beauv. - T., $2 \mathrm{n}=14$., xeromez.-mez., Euras., Bromus sterilis L. - T., $2 n=14$, 28., xeromez., Euras. (submedit.), B. tectorum L. - T., $2 n=14$., xer.-xeromez., Euras. Cont., B. inermis Leyss. - H., $2 \mathrm{n}=28$, 42, 56, 76., xeromez., Euras., $B$. arvensis L. - T.-Ht., $2 \mathrm{n}=14$., xeromez., Euras. (submedit.), B. hordeaceus L. - T.-Ht., $2 \mathrm{n}=28$., mez., Euras. (submedit.), Brachypodium sylvaticum (Huds.) Beauv. - H., $2 \mathrm{n}=14,16,18,28,42,44,56$. , mez.-mezohigr., Euras. (submedit.)., Dasypyrum villosum (L.) P.Candargy - T., $2 n=14$., xeromez., Medit., Taeniaterum caputmedusae (L.) Nevski - T., $2 n=14$., xer., Pont.-medit., Avena fatua L. - T., $2 n=42$., xeromez., Euras., Alopecurus pratensis subsp. pseudonigricans L. - H., $2 n=28$., mezohigr., Euras., Phragmites australis (Cav.) Steud. - G.(HH.), $2 \mathrm{n}=36,48,54,84$, 96., higr., Cosm., Cynodon dactylon (L.) Pers. - G., 2n=36., xeromez., „Delniță”, Cosm., Tragus racemosus (L.) All. - T., $2 \mathrm{n}=40$., xer., Medit., Eragostris minor Host T., 2n=20, 30, 40., xer.-xeromez., „Caracal road”, Centr. Eur.-medit., Sclerochoa dura (L.) Beauv. - T., 2n=14., xeromez., Medit., Digitaria sanguinalis (L.) Scop. - T., $2 n=36$., xeromez.-mez., „Caracal road”., Cosm., Sorghum halepense (L.) Pers. - G., $2 n=20$, 40., mez.- mezohigr., Medit., Dichanthium ischaemum (L.) Roberty - H., $2 n=40,60$., xer.-xeromez., Euras. (submedit.).; Fam. Typhaceae: Typha latifolia L. - G.(HH.), 2n=30., higr., Cosm.

The analysis of the flora identified in the precincts of Celaru commune highlights the xerophilous character of the area. The high proportion held by the xeromesophile species can be explained if a correlation is made with the climatic conditions characteristic for the territory of the administrative unit and of its surroundings (Fig. 2). If the plants that are typical of dry places are added to the above-mentioned species, we can state that $3 / 4$ of the spontaneous flora within Celaru settlement is adapted to a prolonged water stress.

The analysis of bioforms highlights the high proportion held by annual 
species (Fig. 3). This explains the presence of highly extended agricultural surfaces in the area.

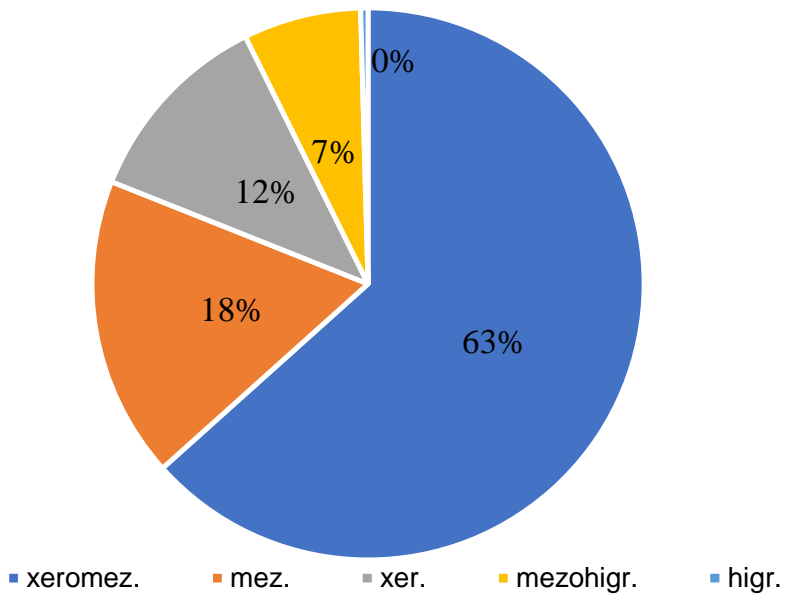

Figure 2. Humidity spectrum (orig.)

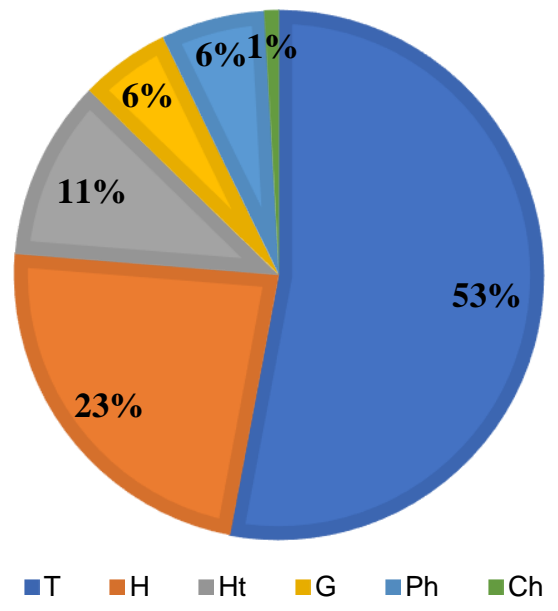

Figure 3. Bioforms spectrum

The presence of phanerophytes is characterized by a very low percentage, which explains the lack of natural arboreal wood vegetation in the settlement and its surroundings. The forests in the area are represented by black locust plantations.

The analysis of geoelements

The analysis of the origin of the vascular plant species from the researched territory underlines the presence of a high proportion of Eurasian species (Fig. 4). This does not bring an element of novelty, as the same situation is also characteristic at national level.

Although the adventitious elements account for a low percentage, they still register a good field representation as specimens, some of them representing invasive species for the Romanian flora. 
The cytotaxonomic analysis shows that out of the total taxa at which the number of chromosomes was found, a number of 106 belong to diploids, 126 to polyploids and the rest is unknown.

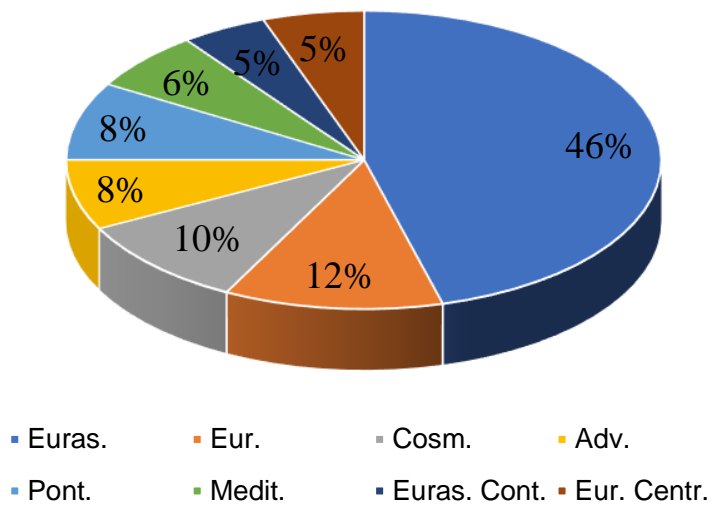

Geoelements spectrum (orig.)

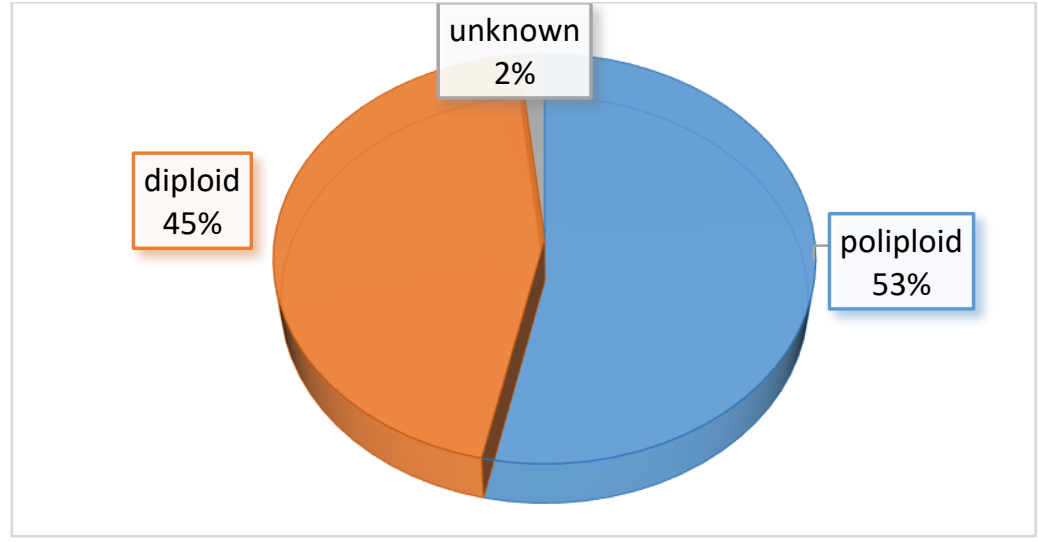

Fig. 4. Cytotaxonomic spectrum (orig.)

\section{The vegetation}

At the beginning of our research in the Celaru area, we tried to make an exhaustive study on the natural and semi-natural vegetation. However, after traveling in the field we found that natural vegetation is almost absent, the seminatural one covers small areas and the anthropogenic one is located at the edge of crops and of ruderal places, as well as and at the level of black locust plantations.

The phytocenoses identified in the field were included in the following associations: Aegylopsietum cylindricae Buia et al. 1959, Amarantho Chenopodietum albi Morariu 1943, Cynodonti-Poetum angustifoliae Rapaics ex Soó 1957, Setario - Veronicetum politae Oberd. 1957, Bromo sterili - Robinietum Pócs 1964. Because these are well-known associations in Romania and, implicitly, in Oltenia, the paper presents the characteristics observed in the Celaru area. 
Aegilopsietum cylindricae Buia et al. 1959.

It forms insular phytocenoses in compacted and very dry areas. The dominant species, i.e. Aegilops cylindrica, is frequently accompanied by: Festuca valesiaca, Poa bulbosa, Dasypyrum villosum, Medicago minima, M. falcata, Filago arvensis, Xeranthemum annuum, etc.

Amarantho - Chenopodietum albi Morariu 1943

Widespread on the borderline of crop fields and especially of the gardens, where the soil is rich in nitrous substances, the association is present in all provinces of the country. Its phytocenoses include a significant number of segetal species, such as: Amaranthus albus, A. blitoides, Hibiscus trionum, which can become co-dominant within the association.

Cynodonti-Poetum angustifoliae Rapaics ex Soó 1957

It is found on plane, dry and sunny surfaces. In addition to the xerophilous species characteristic of the association, numerous ruderal weeds were found in the conducted surveys, which explains the stage of degradation that these phytocenoses display. Among the frequently encountered species we mention: Poa bulbosa, Medicago lupulina, Achillea setacea, Dichanthium ischaemum, Festuca valesiaca and Prunella laciniata.

Setario - Veronicetum politae Oberd. 1957

Phytocenoses of this association were found on the outskirts of the settlement, on the edge and inside agricultural fields. Among the vascular species with higher frequency, we mention: Fumaria schleicheri, Amaranthus retroflexus, Echinochloa crus-galli, Setaria viridis, Chenopodium album, Polygonum aviculare, Sinapis arvensis, Viola arvensis, Cirsium arvense, Polygonum lapathifolium, etc.

Bromo sterilis - Robinietum Pócs 1964 (Syn.: Robinietum pseudacaciae (Arvat 1939) Balász 1942, Agropyro-Robinietum pseudacaciae Szabó 1971)

Black locust plantations are widespread in Oltenia and, implicitly, in the area under study. They are characterized by a very heterogeneous herbaceous flora, with many weeds, such as: Ballota nigra, Anthriscus cerefolium subsp. trichosperma, Urtica dioica, Physalis alkekengi, Galium aparine, Bromus sterilis, Rubus caesius, Leonurus cardiaca, and Alliaria petiolata.

Conservation measures for the vegetation within in the area under study

It has been long believed that nature, through its components, manages to naturally recycle all the atmospheric pollutants that have accumulated in significant quantities.

The key measure for protecting ecosystems is to stop degradation. This degradation is caused primarily by the presence of the human component in nature (Răduțoiu, 2008). This was also observed within Celaru settlement. The vegetation encountered on the territory of this commune and on its borderline areas is subject to a strong zoo-anthropogenic impact, which represents the effect of irrational grazing, domestic waste disposal - especially on the outskirts of the settlement, unsustainable agricultural practices, etc.

\section{CONCLUSIONS}

On the territory of Celaru commune and on its surroundings, there are 236 vascular plants. Among them, there are valuable species from a sociological point of view (e.g. Silene borysthenica), but also allochthonous invasive species (e.g. Chamaesyce maculata, Eleagnus angustifolia, Amaranthus spp., Phytolacca americana, Oxalis fontana, Lycium barbarum, and Ambrosia artemisiifolia). 
Among the identified associations, the best representation is that of Bromo sterili-Robinietum. This is directly connected to the type of sandy soil that is present in the area. The black locust plantations have been and still are used to stabilize land, as this species is one of the few that can withstand the conditions imposed by sandy soil.

The ruderal places, polluted with garbage, are characterized by the presence of phytocenoses framed at Amarantho-Chenopodietum albi, while on the few vineyards in the area we have found Setario - Veronicetum politae.

Certain restoration measures are required in order to restore the vegetal layer on the territory of Celaru commune and on its surroundings; these measures consist in: limiting the extension of the anthropic factor, prohibiting the grazing inside black locust plantations and on the few surfaces covered with grass, which are located on the outskirts of the settlement, and using ecological farming practices.

In conclusion, if urgent protection measures are not taken, we can state that the surface of the settlement under study displays a high and rapid potential of falling into the areas at risk of desertification, which are located in the southern part of the country.

\section{REFERENCES}

Ancuța Gh. Victor et al. 2009. „Celaru, Pagini de istorie locală”

Beldie Al. 1977. Flora României. Determinator ilustrat al plantelor vasculare. Vol. I. Edit. Acad. Rom.. Bucureşti. 406 pp.

Beldie Al. 1979. Flora României. Determinator ilustrat al plantelor vasculare. Vol. II. Edit. Acad. Rom. Bucureşti. 412 pp.

Buia Al. 1959. Există stepă naturală în Oltenia? Soc. Șt. Nat. și Geografie din R.P.R.. Com. Bot.: 93-101.

Buia Al., Popescu-Mihăilă Ana 1952. Contribuţii la flora regiunii Craiova. Buletin Ştiinţific. Secţiunea de Ştiinţe Biologice, Agronomice, Geologice şi Geografice. Tom. IV. nr. 3: 519-536. Bucureşti.

Buia Al. \& Păun M. 1960. Materiale pentru flora şi vegetaţia împrejurimilor oraşului Craiova. SSNG. Com. de Bot., 1957-1959, Bucureşti, 281-296.

Buia Al., Păun M. \& Maloş C. 1961. Pajiştile naturale din Regiunea Craiova şi îmbunătăţirea lor (II). Probleme Agricole. Anul XIII: 31-40.

Ciocârlan V. 2009. Flora ilustrată a României. Pteridophyta et Spermatophyta. Editura Ceres, Bucureşti. $1041 \mathrm{pp}$.

Costache I. 2005. Flora şi vegetaţia bazinului hidrografic inferior al râului Motru. Teza de doctorat. Bucureşti. $290 \mathrm{pp}$.

Păun M. 1963. Materiale pentru flora şi vegetaţia raionului Balş, reg. Oltenia. Lucr. Şt. 6: 35-67.

Păun M. 1964. Flora și vegetația raionului Balș, reg. Oltenia. Rezumatul tezei de doctorat. Craiova. $34 \mathrm{pp}$.

Păun M. 1966. Asociaţiile de buruieni (buruienăriile) din culturile, pajiştile, şi locurile ruderale din Raionul Balş. Regiunea Oltenia. Bul. Ştiinţific Vol. VIII. Institutul Agronomic " T. Vladimirescu ": 27-41. Craiova.

Păun M. 1969. Vegetația pajiștilor de locuri uscate din împrejurimile Balșului. Stud. Cercet. Biol. Ser. Bot. București: 35-44.

Păun M. et Popescu Gh. 1972. Vegetația luncii Jiului dintre Filiași și Zăval (I). Stud. Cercet. Subcom. Mon. Nat. a Olteniei. Cons. Pentru Ocrot. Mon. Nat. Gorj. Extras Craiova: 15-20. 
Păun M. \& Popescu G. 1975. Consideraţii asupra pajiştilor xerofile din Oltenia. Studii și Cercetări. C.C.E.S. Mehedinţi: 95-99.

Sârbu I., Ştefan N. \& Oprea A. 2013. Plante vasculare din România. Determinator ilustrat de teren. Edit. Victor\&Victor, Bucureşti, 1320 pp.

***(http://www.profudegeogra.eu/wp-content/uploads/2011/05/Hartaadministrativa-a-judetului-Dolj.jpg) 